\title{
Impedance Budget and Beam Stability Analysis of the Fermilab Main Injector
}

\author{
M.A. Martens and K.Y. Ng \\ Fermi National Accelerator Laboratory \\ P.O. Box 500, Batavia, Illinois 60510
}

May 1993

Presented at the 1993 Particle Accelerator Conference, Washington, D.C., May 17-20, 1993 


\section{Disclaimer}

This report was prepared as an account of work sponsored by an agency of the United States Government. Neither the United States Government nor any agency thereof, nor any of their employees, makes any warranty, express or implied, or assumes any legal liability or responsibility for the accuracy, completeness, or usefulness of any information, apparatus, product, or process disclosed, or represents that its use would not infringe privately owned rights. Reference herein to any specific commercial product, process, or service by trade name, trademark, manufacturer, or otherwise, does not necessarily constitute or imply its endorsement, recommendation, or favoring by the United States Government or any agency thereof. The views and opinions of authors expressed herein do not necessarily state or reflect those of the United States Government or any agency thereof. 


\title{
Impedance Budget and Beam Stability Analysis of the Fermilab Main Injector
}

\author{
M.A. Martens and K.Y. Ng \\ Fermi National Accelerator Laboratory, ${ }^{*}$ P.O. Box 500, Batavia, IL 60510
}

\section{Abstract}

The impedance budget of the Fermilab Main Injector (MI) is estimated, which includes the contributions from the resistive walls, bellows, if cavities, steps, Lambertsons, etc. Beam stability during ramping and bunch coalescence is analyzed. The transverse resistive-wall coupled bunch growth is found to be somewhat worse than the situation in the Main Ring (MR).

\section{IMPEDANCE AND STABILITY}

One of the important considerations in the design of the Main Injector is the beam coupling impedances in the vacnum chamber and the stability of the beam. Along with the higher intensities comes the possibility of instabilities which lead to growth in beam emittances and/or the loss of beam. This paper makes estimations of the various impedances and instability thresholds based on calculations and measurements. The Main Injector has a mean radius of $R=528.30 \mathrm{~m}$. Its duty is to accelerate a total of $3 \times 10^{13}$ protons in $h=588 \mathrm{rf}$ buckets from the injection energy of $8.9 \mathrm{GeV}$ to the extraction energy of 120 or $150 \mathrm{GeV}$. The threshold for microwave instability has been estimated [1] to be $Z_{\|} / n>20 \Omega$.

\section{A. Resistive Wall}

The shape of the Main Injector beampipe is approximately elliptical with a full width of 4.84 inches and a full height of 2.09 inches. The beampipe is stainless steel, has a thickness of $1.2 \mathrm{~mm}$, and a resistivity of $\rho=74 \mu \Omega-\mathrm{cm}$.

Using the circular approximation [2] to the MI beampipe with a radius of $1.05 \mathrm{in}$, we get the results listed in Table 1. The results are expressed as a function of the revolution harmonic number $n$ for both high and low frequencies. We also approximate the beampipe to the rectangular shape with full height and width of $h=2.09$ in by $w=4.84 \mathrm{in}$. The results can be expressed [3] as the product of a form

\footnotetext{
*Operated by the Universities Research Association, under contracts with the U.S. Department of Energy
}

\begin{tabular}{|c|c|c|}
\hline & $n<n_{c}$ & $n>n_{c}$ \\
\hline$Z_{\|} / n$ & $12.3 / n \Omega$ & $(1+j) 10.2 / \sqrt{n} \Omega$ \\
\hline$Z_{\perp}$ & $19.2 / n \mathrm{M} \Omega / \mathrm{m}$ & $(1+j) 15.4 / \sqrt{n} \mathrm{M} \Omega / \mathrm{m}$ \\
\hline
\end{tabular}

Table 1: Resistive wall impedance as a function of revolution harmonic $n$ for circular beam pipe with radius $b=1.05 \mathrm{in}$. Results are given for frequencies where the skin depth is less (greater) than the thickness of the beampipe $n>n_{c}\left(n<n_{c}\right)$ where $n_{c}=1.44$.

factor times the circular result with radius $b=h / 2$. The form factors are found to be $F_{\|}=0.989, F_{\perp x}=0.404$, and $F_{\perp y}=0.822$. We see that except for the horizontal case, the circular approximation is fairly good.

The most important effect of the resistive wall instability is the transverse coupled bunch instability. Since the growth rate is proportional to $\operatorname{Re} Z_{\perp}\left[(n+\nu) \omega_{o}\right]$ where $\omega_{o} / 2 \pi$ is the revolution frequency, we are interested in the mode $n$ that gives the lowest frequency. In the vertical plane the tune is 25.4 ; so the coupled bunch mode $n=-26$ will have the largest growth rate. The relevant frequency is only $0.6 \omega_{o} / 2 \pi$ which is very small.

Using the low frequency estimates of the Lambertson magnets (see subsection G) and the beampipe, we calculate the growth rate for mode $n=-26$. The beampipe impedance is $32 \mathrm{M} \Omega / \mathrm{m}$ and the Lambertson is $35 \mathrm{M} \Omega / \mathrm{m}$. The growth time of the resistive wall impedance at $8 \mathrm{Gev}$ is then $0.32 \mathrm{msec}$ which is very fast. This is, however, only a bare-bone estimate. Octupoles can cause a tuneshift which is a significant way to lower the growth. Also, with a positive vertical chromaticity, there is a suppressing form factor in the growth formula.

We make a comparison of the growth rate with the calculated value of the Main Ring. Using a current in the Main Ring of 0.115 Amp and a transverse impedance of $97.7 \mathrm{M} \Omega / \mathrm{m}$, the calculated growth rate is $0.65 \mathrm{msec}$. However, experience has shown that with the higher multipole configuration in the Main Ring lattice, the operation chromaticities, together with the dampers, the Main Ring can operate at this intensity without problem. We therefore expect the present Main Ring dampers (or ones with a higher gain) to be sufficient to damp the transverse resistive wall instability in the Main Injector. 


\section{B. Space Charge}

For the space charge impedance estimation, we make estimates based on beampipes with circular and rectangular cross sections. The circular cross section results are well known while those for the rectangular case come from conformal mapping techniques [3].

Using the formula for the circular and rectangular cross sections, the space charge impedance was calculated at $8 \mathrm{Gev}$ for a beam with a total $3 \times 10^{13}$ particles and $30 \pi \mathrm{mm}-\mathrm{mr} 95 \%$ normalized emittance. With $\beta_{\min }=10 \mathrm{~m}$, this corresponds to a cylindrical beam with a radius of $a=5.6 \mathrm{~mm}$. The results are shown in Table 2. The space charge impedance is important in contributing to the negative-mass instability at transition and for mismatch of the equilibrium bunch length before and after transition. These issues are not considered in this paper.

\begin{tabular}{|c|c|c|}
\hline & $\begin{array}{c}\text { Circular } \\
\text { Beampipe }\end{array}$ & $\begin{array}{c}\text { Rectangular } \\
\text { Beampipe }\end{array}$ \\
\hline$Z_{\|} / \mathrm{n}$ & $-j 7.98 \Omega$ & $-j 9.61 \Omega$ \\
\hline$Z_{\perp x}$ & $-j 67.0 \mathrm{M} \Omega / \mathrm{m}$ & $-j 68.4 \mathrm{M} \Omega / \mathrm{m}$ \\
\hline$Z_{\perp y}$ & $-j 67.0 \mathrm{M} \Omega / \mathrm{m}$ & $-j 67.6 \mathrm{M} \Omega / \mathrm{m}$ \\
\hline
\end{tabular}

Table 2: Space charge impedance for both circular and rectangular beampipe. Calculations were made at $8 \mathrm{Gev}$ using a cylindrically uniform beam with transverse radius of $5.62 \mathrm{~mm}$.

We are also interested in the coherent and incoherent tune shifts due to the electric image charges in a rectangular beampipe [3]. For bunched beam at $8 \mathrm{GeV}$ with bunch length of 150 degrees the coherent tune shift in the vertical direction is -0.13 and in the horizontal and vertical directions the incoherent tune shift is \pm 0.043 .

Also the incoherent tune shift due to the beam itself was calculated for a round Gaussian beam with a radius of $5.62 \mathrm{~mm}$. The value was found to be 0.014 for the bunched beam. It is possible that the total incoherent tune shift of 0.057 in the horizontal direction might be too large and pose a problem in operation.

\section{RF Cavities}

Since the Main Injector RF cavities will be transferred from the Main Ring, previous measurements of the cavities can be used for the estimation of the longitudinal impedance. The measurements on the cavities were made using a stretched wire technique [4] and the results are shown in Table 3. The mode at $128 \mathrm{MHz}$ has limited Main Ring performance in the past so a mode damper was designed and installed [5].

We calculate the coupled bunch growth times due to the $R F$ cavities. The nominal parameters for the Main injector are: particles per bunch is $6 \times 10^{10}$, the RF voltage is 400 $\mathrm{kV}$, and the bunch area is $0.25 \mathrm{eV}$-s. As a comparison, we also calculate the growth rates in the Main Ring. The growth times are given in Table 3 for both the Main Ring

\begin{tabular}{|c|c|c|c|c|c|}
\hline \multirow{2}{*}{ Freq $(\mathrm{MHz})$} & \multirow{2}{*}{$Z_{\|}(\mathbf{k} \Omega)$} & \multicolumn{3}{|c|}{ growth time in msec } \\
\cline { 3 - 6 } & & \multicolumn{2}{|c|}{ dipole } & \multicolumn{2}{|c|}{ quadrupole } \\
\cline { 3 - 6 } & & MI & MR & MI & MR \\
\hline 71.0 & 10.0 & 16.8 & 45.0 & 280 & 500 \\
100.0 & 2.5 & 53.4 & 151.9 & 440 & 810 \\
128.0 & 6.3 & 19.2 & 59.1 & 93 & 180 \\
223.0 & 111.0 & 1.4 & 6.8 & 1.8 & 4.6 \\
600.0 & 277.0 & 5.1 & 30.0 & 2.8 & 12 \\
850.0 & 49.0 & $\mathbf{5 3 . 2}$ & 321.4 & 38 & 140 \\
\hline
\end{tabular}

Table 3: Dipole and quadrupole mode growth times for both the Main Injector and the Main Ring due to the longitudinal of cavity modes.

and the Main Injector. For the Main Ring we used $3 \times 10^{10}$ particles per bunch.

Experience with the Main Ring has shown that the 128 $\mathrm{MHz}$ mode can sometimes be a problem in fixed target operation. Also of concern is the short growth time of the 223 $\mathrm{MHz}$ mode. As a result, a set of passive dampers for the cavities is being designed to reduce the shunt impedance of the $223 \mathrm{MHz}$ mode and further damp the $128 \mathrm{MHz}$ mode as well. With this mode damper installed, the $223 \mathrm{MHz}$ impedance is lowered to a value of about $10 \mathrm{k} \Omega$.

Also of concern is the coupled bunch instability with short batches used in coalescing since standard coupled bunch theory is not applicable to a partially filled ring. Presently in the MR there is evidence of an instability with $3 \times 10^{10}$ particles per bunch. Since this type of instability depends on $R / Q$ and not on $R$, adding passive dampers will not remove or improve the instability. In the Main Injector there will be $6 \times 10^{10}$ particles per bunch so there could be substantial difficulty with the short batch operation. The problem will likely require the development of an active damping system.

The growth times for the Main Injector are about one half of those in the Main Ring (Table 4). So far there has not been any evidence of transverse coupled bunch mode observed in the Main Ring.

\begin{tabular}{|c|c|c|c|c|c|}
\hline \multirow{2}{*}{ Freq $(\mathrm{MHz})$} & \multirow{3}{*}{$Z_{\perp}(\mathrm{M} \Omega / m)$} & \multicolumn{4}{|c|}{ growth time in msec } \\
\cline { 3 - 6 } & & \multicolumn{2}{|c|}{ dipole } & \multicolumn{2}{c|}{ quadrupole } \\
\cline { 3 - 6 } & & MI & MR & MI & MR \\
\hline 398 & 3.3 & 5.1 & 9.0 & 5.2 & 9 \\
454 & 1.9 & 9.9 & 17 & 20 & 18 \\
566 & 0.75 & 32 & 56 & 32 & 57 \\
700 & 0.13 & 230 & 410 & 230 & 410 \\
1260 & 0.18 & 290 & 510 & 290 & 520 \\
1270 & 1.7 & 31 & 54 & 31 & 54 \\
1290 & 2.4 & 23 & 40 & 23 & 40 \\
\hline
\end{tabular}

Table 4: Dipole and quadrupole mode growth times for both the Main Injector and the Main Ring due to the transverse rf cavity modes. 


\section{Beam Position Monitors (BPM)}

The Main Injector BPM consists of 4 stripline pickups which are cut out of the approximately elliptical beampipe. Two pickups are located on each of the top and bottom surfaces of the beampipe and are spaced $40 \mathrm{~mm}$ apart from center to center. Each stripline is about $1 \mathrm{~cm}$ wide, $\ell=40 \mathrm{~cm}$ long, and has a characteristic impedance of $Z_{s}=50 \Omega$. The downstream end of each stripline is shorted while the upstream end is connected in parallel with a $50 \Omega$ cable which leads to the RF module.

As a bunch passes the BPM, only a tiny fraction of the image current on the beampipe is picked up by the stripline. We estimate this fraction to be $f=0.055$ and then calculate the power absorbed by the striplines. Since this is the same as the power lost by the beam, we can calculate the beam impedance. For a set of four striplines,

$$
Z_{\|}=2 f^{2} Z_{s}\left[1-\cos \left(\frac{2 \ell \omega}{c}\right)+j \sin \left(\frac{2 \ell \omega}{c}\right)\right] .
$$

For 208 sets of BPM in the ring, $Z_{\|} / n=0.050 \Omega$ below $\sim 60 \mathrm{MHz}$, then starts oscillating and rolls off. In the same way, the transverse impedances of the BPM can also be computed. They have the same frequency dependency as $Z_{\|} / n$. For example, the vertical impedance at low frequencies is $Z_{\perp y}=259 \mathrm{k} \Omega / \mathrm{m}$. These impedances are much lower than those of other components in the ring. This is mainly due to the relatively narrow width of the stripline pickups. Thus the BPM's are not expected to be a problem.

\section{E. Beam Valve}

Although the beam valve design is not yet completed, its general shape is known. Using this general shape, the 3D computer code MAFIA [6] was used to find the longitudinal and transverse impedances. The beam valve is assumed to have the same resistivity as the beampipe, $\rho=74 \mu \Omega$-cm.

Calculations for the coupled bunch growth rates show that most of the beam valve modes are benign. This is mainly due to the fact that the cavity modes are at frequencies which are above the beam spectrum frequencies. All modes have a growth time greater than $50 \mathrm{~ms}$ except for the quadrupole mode of the $2.46 \mathrm{GHz}$ mode which has a growth rate of $38 \mathrm{~ms}$.

\section{F. Bellows}

The beampipe bellows will consist of approximately 10 to 17 convolutions extending over one inch. The inner dimension of the bellows is an ellipse of similar size to the beampipe and the convolutions extend 0.5 inches. To estimate the impedance the elliptical shape was approximated by a circular beampipe with radius 1.05 inches. This was done for both 10 and 17 bellows convolutions using the program TBCI [7].

The longitudinal impedance is very similar for both the 10 and 17 bellows case. The impedance peaks at $120 \Omega$ near
$8.5 \mathrm{GHz}$. For 516 bellows in the Main Injector, we have a total of $Z / n=0.65 \Omega$ at $8.5 \mathrm{GHz}$. At low frequencies, it is $Z / n=j 2.8 \Omega$. According to previous calculations this impedance is below the threshold for the Main Injector except near transition [1].

\section{G. Lambertsons}

The main concern of the Lambertson magnets is the low frequency component created by the exposure of the beam to the bare laminations of the magnet. A rough estimation of the Lambertson magnets is made by approximating the magnet as a series of annular laminations of $0.953 \mathrm{~mm}$ width. The inner radius is chosen to be $b=2.54 \mathrm{~cm}$ and the outer radius is $d=5.08 \mathrm{~cm}$. We use a resistivity of $\rho=$ $20 \mu \Omega-\mathrm{cm}$ and a permeability of $\mu=100$ for the lamination material. The impedance is estimated by integrating the resistance of the low frequency current traveling through the laminations.

With 25.8 meters of Lambertson magnets in the Main Injector the total low frequency resistive wall impedance is calculated to be

$$
\frac{Z_{\|}}{n}=(1+j) \frac{16.4}{\sqrt{n}}
$$

And to estimate the transverse impedance, we use the approximate relation

$$
Z_{\perp}=\frac{2 c}{b^{2}} \frac{Z_{\|}}{\omega}=(1+j) \frac{26.8}{\sqrt{n}} .
$$

It should be noted that the Lambertson magnet was assumed to have a circular geometry with inner radius of $b=2.54 \mathrm{~cm}$. The actual shape of the Lambertson is much different, so this estimate can only be approximate. Using a slightly larger inner radius can change the impedance, especially the transverse one, by a significant amount (i.e. if $b$ is $10 \%$ larger the impedance drops by $25 \%$ ).

\section{REFERENCES}

[1] King-Yuen Ng, Fermilab Main Injector Note MI-0029.

[2] B. Zotter and F. Sacherer, CERN 77-13, pp. 175-218, (1977).

[3] K.Y. Ng, Particle Accelerators, 16, pp. 63-80, (1984).

[4] R.A. Dehn, Q.A. Kerns, and J.E. Griffin, "Mode Damping in NAL Main Ring Accelerating Cavities", IEEE Trans. Nucl. Sci., NS-18, pp. 260-261, (1971).

[5] Q.A. Kerns and H.W. Miller, "Fermilab 500-Gev Main Accelerator RF Cavity 128- MHz Mode Damper", IEEE Trans. Nucl. Sci., NS-24, pp. 1704-1706, (1971).

[6] DESY, LANL and KFA, MAFIA User Guide, (1989).

[7] R. Klatt and T. Weiland, TBCI Short User Guide, DESY, (1988). 